\title{
Rethinking Wearable Activity Trackers as Assistive Technologies: A Qualitative Study on Long-Term Use
}

\author{
Lin $\mathrm{Li}$ \\ Michigan State \\ University \\ lilin6@msu.edu \\ Wei Peng \\ Michigan State \\ University \\ pengwei@msu.edu
}

\author{
Anastasia Kononova \\ Michigan State \\ University \\ kononova@msu.edu
}

\author{
Kendra Kamp \\ University of \\ Washington \\ kamp@uw.edu
}

\author{
Shelia Cotten \\ Clemson \\ University \\ scotten@clemson.edu
}

\begin{abstract}
This study proposes that wearable activity trackers (WATs), such as Fitbit, Apple Watch, can be viewed as assistive technologies to promote older adults' health and independent living. Qualitative interview data with 20 older adults (65 and older) who had used WATs for six months or longer were analyzed within the framework of the Match Person and Technology (MPT) model. We found that personal and psychosocial factors, environmental factors, and technology-related factors contributed to the participants' long-term engagement with WATs. Determination and self-discipline, support from one's family members and friends, and goal setting and feedback of goal accomplishment were among the most mentioned facilitators of using WATs for more than six months. We discussed the design implications of these findings.
\end{abstract}

\section{Introduction}

Wearable activity trackers (WATs) have shown great potential in promoting older adults' physical health [1$3]$. For example, a 12 -week study $(N=34)$ evaluated trackers' feasibility and utility among older adults and found that $95 \%$ of the participants achieved a reduced waist circumference and increased step counts [3]. A randomized controlled trial $(N=51)$ showed that, compared to a group of older women using a pedometer, another group of female participants using a web-based Fitbit was significantly more active [1]. One consistent issue plaguing WAT usage is the lack of long-term engagement with these devices. Long-term use may be especially hard to achieve with the older adult population, given that the older adults are known to feel less comfortable, have less experience, and have lower self-efficacy and perceived control over the usage of information technologies $[4,5]$. Several studies have investigated the reasons for abandonment [6-9] while leaving a gap in examining reasons for long-term use. Theoretically driven research that organizes factors that may promote the long-term use of WATs among older adults is also lacking $[6,9]$.
Assistive technologies are devices adopted by people with disabilities to cope with various tasks in their everyday lives and preserve their access to the environment, technology, information, and services [10]. WATs can be viewed as a form of assistive technology in its purpose of monitoring, regulating, and promoting healthy behaviors that are conducive for the positive functioning of older adults in the long-term [11-14]. As mobility, popularity, functionality, and processing speed of new mobile technologies allow for more efficient and effective means to monitor health, it will enable a brand-new vision of what daily life can look like for older individuals and individuals with disabilities [11]. In proposing a framework that extends at home (@home) assistive technologies, scholars advocated for viewing pervasive sensor devices, such as WATs, as forms of assistive technologies that help vulnerable populations to remain living at home independently as long as possible [11]. However, similar to the attrition with WATs, about $20-30 \%$ of assistive technologies, such as mobility aids, are discarded within a year after acquisition [15-17]. In thinking about how WATs can become useful for older adults, we draw insights from a comprehensive framework about the adoption and continued use of assistive technologies: The Match Person and Technology (MPT) model. The MPT model identifies important factors that predict the use/non-use of assistive technologies and their subsequent match with the individuals' needs at a three to four month followup, which is equivalent to the continued usage of assistive technologies [18, 19]. The model was developed using the grounded theory approach. It proposes three types of predictors for assistive device use or abandonment: a) personal and psychosocial, b) environmental, and c) technology-related factors.

\section{Background literature}

The study of WATs has mostly been independent of research on assistive technologies. Studies on WATs focus on their acceptability and usability across various populations $[3,20,21]$, their validity or reliability in measuring a variety of activities [22], factors associated with their adoption and abandonment [23-26]. In the 
meantime, research on assistive technologies seeks to inform better design solutions for older adults in tracking their mobility and activity data with a lack of consideration on how these design solutions match the specific needs and constraints of older adults in a reallife setting $[27,28]$. As a theoretical framework that has been tested empirically, the MPT model brings together a host of factors related to the acceptance and adherence of different assistive technologies. By adopting the MPT model, this study investigates how similar personal, psychosocial, environmental, and technological factors contribute to older adults' decision-making process to use WATs on a long-term basis.

In the MPT model, personal and psychosocial predispositions (e.g., attitudes, mood, motivations, autonomy, self-determination, self-esteem, sense of control, and readiness for technology use) have been found to be positively associated with assistive technology use and continued use during the three to four month follow-up [18, 29, 30]. Environmental factors in the MPT model refer to physical, social, and attitudinal environments surrounding an individual's assistive technology use, such as funding support for technology, technical support and training, and general reactions of others toward technology use [23]. Environmental factors also include focusing on users themselves during technology selection, family/peers/employer support, and being in settings/environments that encourage the use or make use easy or comfortable [18]. Specifically, having professionals who can help with assessing individuals' needs, priorities, and preferences thoroughly in selecting and obtaining assistive technology and who acknowledge that individuals' needs change over time as their disability develops are also considered to be environmental facilitators [29, 30]. Lastly, the MPT model defines technology-related factors as technology's physical and cognitive demands, sensory requirements, cost, training, repair and maintenance issues, aesthetics, as well as the specific functions, features, and usability of the assistive device [29]. Device usability related to its size, weight, durability, and others may be additional factors in influencing users' subjective judgments about its "useworthiness," i.e., the perception that the technology has to be worth using to be utilized.

We argue that the three sets of factors mentioned above can serve as a useful framework for understanding the continued use of WATs among adults who are 65 or older, based on evidence supporting adults' adherence to physical activity, which is the goal for using WATs. For example, on the personal level, psychological factors, including self-regulation and self-efficacy, are the most identified predictors of weight loss [31]. One study identified that intrinsic motivation is related to different levels of adherence to activity tracking [32]. On the level of the environment, social support is related to individuals' engagement with physical activities [33-35]. With regard to technologyrelated factors, WATs incorporate validated behavior change techniques (BCTs), among which are selfmonitoring, self-regulation, feedback on performance, social comparison, social support, and goal setting [36, 37]. In viewing WATs as a form of assistive technology that promotes the quality of life and independent functioning of older adults, we are interested in answering a more specific question for understanding its long-term usage based on the MPT model:

RQ: What specific a) personal and psychosocial factors, b) environmental factors, and c) technologyrelated factors contribute to continued use of WATs among older adults $65+$ ?

\section{Method}

\subsection{Participants}

We recruited WAT users via surveying a Qualtrics panel of older adults aged 65 and older. Three-hundredand-fourteen individuals finished the survey, and 163 of them reported that they had used WATs for more than six months, which we defined as long-term users based on previous literature [38-40]. Among the 163 longterm users, 71 were interested in our follow-up study. We randomly chose 20 out of the 71 for the in-depth interview. The average age of the sample was 67.95 years old $(\mathrm{SD}=2.01)$. Fifty-five percent of the sample was female. Older adults in this sample had used WAT for an average of 31.9 months at the time of the interview $(S D=25.64)$.

\subsection{Procedure}

We contacted the 20 participants via phone first to confirm their interest in interview participation. All participants agreed to participate in a one-hour interview. At the end of the first interview, we asked participants to use their smartphones to take pictures for a week to provide insights into the role of WATs in their daily lives. The participants texted or emailed the photos with annotation to the research team. We asked participants to use these images during the follow-up interview to help them recall details of their WAT usage. After completing the first interview, participants received a \$30 Target, Walmart, or Amazon gift card via mail or email. After completing the photo-taking exercise and the second interview, participants received a \$50 gift card to the store of their choice. This study reports the parts of the study that pertain to the personal and psychosocial factors, environmental factors, and technology-related factors related to long-term use. 


\subsection{Interview Materials}

We conducted semi-structured interviews. For the first interview, researchers began with obtaining consent. Once consent was provided, we asked the ice breaker questions, including the following, "Can you describe how you use your tracker on a typical day, and how often do you wear your tracker?" Next, we asked questions that were designed to be general and exploratory so participants could talk freely about their experiences with WATs. From their answers, we later identified the personal, environmental, and technological factors that prompted their WATs usage. Participants were first asked to tell the story of when they first started using their tracker; to identify reasons to start using one, reasons for selecting a particular type of tracker, and reasons for their continued usage of the tracker; to describe WAT features they used the most and least and why, and to give advice they would give to a person who would like to become a long-term WAT user. To refresh participants' memory, the interviewer began the second interview by asking participants to look at each photo of WAT usage that they previously submitted. After asking contextual questions, such as "Where were you when you took this photo?", the interviewer asked follow-up questions that specifically focused on the physical, social, and mental benefits of WATs. Lastly, the participants were asked about the successful strategies to continue sustainably using the WATs that were captured in their photos.

\subsection{Data Analysis}

We used an online transcription service to record and transcribe the two rounds of interviews verbatim. We performed inductive data analysis and identified common nodes via exploratory thematic analysis. Initially, four researchers iteratively analyzed three randomly selected transcripts to generate nodes using the NVivo 10 and NVivo 11. After establishing a consensus over the main nodes with the three interview transcripts, two researchers analyzed nine transcripts with odd-number participant IDs. The other two coders analyzed eight transcripts with even-number participant IDs. Then the researchers reviewed, discussed, and resolved any disagreements over coding. A third researcher was invited to address the dispute if the two researchers could not agree on the coding of the same transcript. The nodes we derived during the initial round were revised throughout the coding process. Further, applying the MPT theoretical framework, we identified and reported themes relevant to our research question based on the final nodes.

\section{Results}

Within the theoretical framework of the MPT model, the research question explored what personal and psychosocial, environmental, and technologyrelated factors contributed to continued WAT use among older adults aged 65 and older.

\subsection{Personal and Psychosocial Factors: Determination and Self-Discipline}

Echoing the prediction of the MPT model, our interviewees cited determination and self-discipline as predisposing factors for WAT use continue passed six months. Many participants emphasized that no matter how well-equipped individuals were with technology or facilitating social or physical environments, ultimately, long-term use depended on the mindset of the individuals. They believed that only those who were determined and disciplined could carry out the action of putting on the WAT every day, monitoring and changing behaviors accordingly, and not giving up even when facing challenges.

"It's all self-motivation, and the tracker helps you see how you're doing. You have to make the improvements, not the tracker." [Participant 3, male, 67 years old]

"I'm kind of a goal-oriented person and when I make up my mind to do something, I'm gonna do it. I made up my mind at the start that I was gonna wear the tracker and that I was gonna see the results and so that's what my thinking was from the very start was 'Okay, we're gonna do this and we're gonna keep up with it.' " [Participant 4, female, 68 years old]

'I'm just wired that way. I grew up with the military dad, so I guess that's the reason why it's just ingrained in me to whatever you start, you finish...... I was determined that I was not going to do that." [Participant 9, male, 68 years old]

"For the health benefits. I think I'm a very disciplined person, so I know that's why I keep doing this. I know a lot of people aren't, especially younger people, they just aren't real disciplined. If I don't have the 10,000, like I said, or the 30 ...Active minutes on it, I'm outside till I get it." [Participant 7, female, 68 years old] 


\subsection{Environmental Factors: Family, Friends, and Doctors}

Although long-term users considered their internal motivation and self-discipline to be the essential reasons for continued WAT use, they also acknowledged that external facilitators made the process easier. Family members and friends were the most frequently discussed external facilitators. Some participants created challenges with family members and friends to determine who could obtain the most steps. The WAT also facilitated inter-generational connections as older adults were able to connect with grandchildren by comparing steps. A good portion of the participants received their WAT as a gift from family members and friends, which initiated their use. The support of family members and friends also helped participants overcome barriers and provide the motivation to maintain use. Although not all participants connected to other WAT users to engage in competition, some participants found friendly matches with friends, family members, and even strangers, motivating.

"My son, he gets in there and gives me those, you know, I don't know if you know on the Fitbit website, they have it where you can make faces. And when I don't do as good as that he sends me a face that's sticking out its tongue....... It's good to have a competitor partner, somebody that I compete with because it keeps you ... I think anything you can do with somebody else is easier to do than by yourself. The Fitbit is one of the things you can do by yourself but bring other people into it on the internet. You don't have to go out and walk with someone, because you're still competing with somebody that you don't have to be with." [Participant 12, female, 70 years old]

Besides the direct encouragement from or engagement with one's family members and friends, participants also frequently mentioned how their doctors' recommendations about improving their physical health prompted their WATs use on a longterm basis. In relation to what the MPT model posits about settings/environments that encourage assistive technology use, recommendations by one's doctors to increase physical activity indirectly contributed to participants' adherence to WATs.

"How it all started is I have [inaudible] from Vietnam and my pancreas is shut down. I have a lot of health issues, so the doctor said, 'Probably the best thing you can do is walk.'
I started walking 15 miles every day. ...... It's been a real progress. Matter of fact I'm just looking at my lifetime here, it's 24,130,826 steps on my Fitbit." [Participant 19 , female, 70 years old]

"The other thing that really got me going on this thing was when I went to see her [the doctor-authors] in February and she did all my blood work, last year, my A1C was above the range it should have been. Not high, but she said, 'If this continues, you're on the pre-diabetes.' She really hit on me about my exercising and so forth...... because once I retired, I started to do some walking but I wasn't real serious into it. She laid it out to me there, end of February. 'You're going to have a choice. We're going to give you injections, or it's borderline exercise, and eating right can reduce it." 'Participant 20 , male, 68 years old]

\subsection{Technology-related Factors: Goal Setting and Feedback}

The participants used various brands and models of WATs with different features. The most prominent features that promoted continued use were goal-setting and feedback on goal accomplishment. This also relates to personal factors such as self-discipline because the goals set on WAT by the participants served as a measurable target for them to focus on. Participants also mentioned they did not always follow the default goal suggested by the device and, instead, set realistic goals based on their conditions and gradually modified their goals to become more active.

"I look at it and I'll go I've got 2.95 miles, and I'm going, oh gosh, okay, let's see how many times I can walk back and forth in front of the house before I get to three. It's one of those things that if you didn't have it, you wouldn't do that. You'd say oh I think I've walked this amount of miles. I'm good." [Participant 3]

"The biggest thing about any of these is just wearing them and using them, and once they're set up properly, it's going to tell you whether you're achieving your goals. That's the other thing, you have to determine what your goals are." [Participant 6 male, 66 years old] 
Additionally, feedback showing completion of goals boosted confidence and intrinsic motivation. Each round of goal setting and goal accomplishment increased participants' confidence and self-efficacy of using WAT to monitor physical activity. It likely helped them form a habit and become long-term users.

"I think that probably the best that can happen is that you see the results. And when you see the result and the results are the ones you want or towards the ones you want makes you excited. Okay. Oh, cool. I'm getting somewhere and seeing that this is happening, so this is happening to me and encourage me to keep going." [Participant 10 , female, 67 years old]

"My goal is to do 10,000 steps each day if possible. That's not always ... I don't always achieve that, but that's the target at least. That way, by having it on with me at all times during the course of the day, by the end of the day I can gauge whether I've met that goal that day. I would say probably maybe 70 percent of the time I meet that goal, but again it's not every day by any means. That way it gives me an idea whether I need to add activities through the course of the day. If I'm having a week where I'm not meeting my target as well as I'd like to, then I try to [maintain - authors] my activity level so that I get closer to the goal that I'm seeking." [Participant 18, male, 65 years old]

Besides these features embedded in WATs, participants also expressed that the mere existence of the technology served as a gentle reminder of what they had planned to achieve for improving their health. Thus, WATs were used as a cue for self-monitoring and for taking action to modify participants' health-related behaviors.

"Like when people go to counseling and they know they're going to have to talk to their counselor. They're spending money for it. They're more likely to follow through what a counselor will tell you instead of it just being oh, if I think about it, I'll do it. Now I think the Fitbit being on your wrist would be enough to be that gentle reminder as well." [Participant 09, male, 68 years old]

"[If - authors] I didn't have the tracker, it would certainly be much more difficult for me to get into that mindset of: 'Okay, I need to pick up the pace. I need to do something more active because I'm falling into my old routine where I really wasn't quite as active.' This way it really is a trigger that says: 'Okay, it's time to pick it up a little bit.' It's very helpful from that perspective." [Participant 18, male, 65 years old]

\section{Discussion}

Driven by the theoretical model of MPT, this study proposed that WATs could be reconsidered as a form of assistive technology for older adults. We analyzed qualitative data related to personal and psychosocial, environmental, and technology-related factors that contribute to the long-term use of WATs. The findings of this study not only offer insights into the ways to promote long-term use of WATs among seniors but also have design implications for other health information technologies targeting sustainable behavior change. The results of this study suggest that any interventions designed to increase WAT use and physical activity among older adults will need to take into account a range of factors. The study will also inform strategies to facilitate the successful development of the WAT use habit among older adults.

\subsection{Personal and Psychological Factors}

Following the MPT model that highlighted autonomy and self-determination as personal factors that affect the uptake and continued usage of assistive technologies for individuals [19], we found that our participants identified self-discipline as one determining factor for their continued use of WATs. Although the literature on long-term use of personal informatics devices is lacking, numerous research of continued behavior modification in health, such as weight loss and physical activity (which are often the goals of using WATs), has shown self-regulation as one of the significant psychological predictors for engaging in prolonged behavior changes [31, 41, 42]. Like in any technology-assisted behavior modification, individual differences may modify the effectiveness of sustainable WAT usage. By identifying personal and psychological factors that moderate the effectiveness, a possible solution might be to provide additional interventions to leverage individuals' predispositions and maximize their personal motivations to stay healthy by utilizing WATs. Designers of WATs and other personal informatics devices that intend to encourage prolonged use may also consider incorporating features that support self-regulation. 


\subsection{Environmental Factors}

The MPT model outlined family/peers/employer support as an environmental facilitator that positively affects the long-term use of assistive technologies [18]. Following the model, we identified that support from family, friends, and health care providers contributes to the continued use of WATs. This result is consistent with the evidence that technological features addressing social connections are considered to be one of the most helpful features to encourage physical activity among older adults [43]. The support from family, friends, and physicians was related to maintaining physical activity, albeit not necessarily directly related to wearing a WAT. However, by wearing a WAT, the participants demonstrated concrete evidence of step counts to their family members, friends, and, potentially, doctors. Additionally, wearing WATs allowed participants to engage in a friendly competition of step counting with family members and friends.

The other way social support can impact the use of WATs is demonstrated by the digital divide literature in which individuals' social networks provide valuable technical assistance and an encouraging environment for individuals to learn how to use digital technologies, including WATs [44-46]. The MPT model emphasizes the help of professionals in selecting and maintaining the use of assistive technologies. In the context of using WATs, this can be evidenced by the help of family members and friends in figuring out how to use specific technology functions and features and solving technical issues that occur during WATs use. The finding suggests that designers of personal informatics devices should consider features that increase opportunities to engage in social interaction with close social ties for prolonging WATs use.

\subsection{Technology-related Factors}

Among available WAT features, goal setting and feedback were the two most mentioned helpful features. Goal setting and system rewards features are frequent in most WATs. For example, the Fitbit tracker provides a default goal of 10,000 steps a day and allows customization of personal goals. Additionally, the Fitbit website integrates a system of achievement badges that are awarded to those who meet specific criteria such as walking 30,000 steps or reaching a walking distance of 500 miles [47]. Nevertheless, the default goal of 10,000 is not always feasible for older adults, especially those with preexisting health conditions. One common strategy that our participants adopted was to adjust to the goals that fit with their current physical capability. As they made progress towards achieving realistic goals, they set higher objectives to fulfill their potential. Receiving positive feedback in the form of daily quantified steps was also an important motivator. In displaying users' effort in achieving their goals, various strategies such as bar charts [48] and graphic representations are used by designers [43]. Since studies have reported that users prefer positive over negative feedback [48, 49], most persuasive technologies display positive progress to users [50]. Besides technology features inherent in WATs, the presence of WATs also reminds older adults to put consistent efforts into maintaining their health.

In light of these findings, designers of wearable devices for older adults may consider the specific and frequently occurring health conditions associated with this population and, therefore, facilitate a steadily increased regimen of step count goals. When seniors begin using the WAT, it may be beneficial to set up such personalized regimens by surveying them about their age, activity level, and health status to prescribe realistic goals and adapt the goals based on synchronous data from the user.

\section{Limitations and future research}

This study has several limitations. First, besides the MPT model, other theoretical models, for instance, the expectation-confirmation model, could be used to predict the continued use of technology [51], which have already been adopted in research regarding the use of WATs and other health informatics devices [52]. Second, more questions regarding how older adults obtain their WATs, i.e., through doctor's or friends' suggestions, how they paid for their WATs, as well as their opinions on the direct comparisons regarding WATs and assistive technologies could be added. These questions could contribute to our argument in viewing WATs as assistive technologies. Third, in our interview, some participants anecdotally mentioned their other chronic illnesses and acute health crises that prompted or sustained their WATs usage. However, we did not systematically document the morbidities that accompanied older adults' long-term WATs use. This gap should be addressed in future studies to strengthen the argument that the most vulnerable patients could greatly benefit from using WATs as an assistive technology to regulate their health behaviors and improve their health. Future studies should explore the possibilities of designing and using WATs to improve health of those who suffer from specific types of chronic and acute diseases.

\section{Conclusion}

WATs have been heavily marketed as new and "cool" gadgets for the younger populations. However, shifting perspectives in viewing WATs as assistive 
technologies help guide the study in identifying factors that contribute to older adults' successful use of WATs. When there is a shared understanding of these contributing factors, the usability issues as well as the acceptance and adherence issues are more likely to be systematically addressed with a concerted effort from both WATs and assistive technologies researchers.

As the aging process is usually accompanied by illnesses that hinder physical mobility, WATs, as a device designed to encourage physical mobility through integrating behavior change techniques and social support, have great potential in promoting and maintaining the health of aging individuals [53, 54]. Despite its demonstrated effectiveness in improving physical activity levels among sedentary older adults [55], there is still significant negativity, frustration, and anxiety toward assistive technologies and health information technologies among older adults [14, 56]. Other barriers related to data inaccuracy, lack of adequate instructions [24], device characteristics such as longevity, ergonomics, and aesthetics, as well as financial costs [57], are also identified by researchers that examined older adults' acceptance of WATs. The older adult population has a higher prevalence of chronic conditions as well as a lower level of adopting digital trackers, compared with their younger counterparts [52]. Nevertheless, there is also a considerable amount of interest among older individuals to monitor their health indicators [52]. When WATs are used in conjunction with other mHealth and eHealth technologies, including mHealth Apps, electronic health records, smart medical devices (e.g., blood pressure/glucose monitors), they can provide constant and comprehensive monitoring of the health measures of the older adults and connect them with caregivers and health care providers in cases that need intervention. The findings of this study provide insights regarding how to promote using WATs on a long-term basis; support the independence of older individuals living at home; increase their caregivers' and health care providers' regular access to activity information; and improve older adults' quality of life [58, 59].

\section{References}

[1] L. A. Cadmus-Bertram, B. H. Marcus, R. E. Patterson, B. A. Parker, and B. L. Morey, "Randomized trial of a Fitbit-based physical activity intervention for women," Am J Prev Med, vol. 49, no. 3, pp. 414-418, Sep. 2015, doi: 10.1016/j.amepre.2015.01.020.

[2] K. Mercer, M. Li, and K. A. Grindrod, "Do wearable activity trackers have a place in pharmacies?," Canadian Pharmacists Journal / Revue des Pharmaciens du Canada, vol. 148, no. 3, pp. 134137, May 2015, doi: 10.1177/1715163515579221.

[3] T. O'Brien, M. Troutman-Jordan, D. Hathaway, S. Armstrong, and M. Moore, "Acceptability of wristband activity trackers among community dwelling older adults," Geriatric Nursing, vol. 36, no. 2, pp. S21-S25, Mar. 2015, doi: 10.1016/j.gerinurse.2015.02.019.

[4] S. J. Czaja et al., "Factors Predicting the Use of Technology: Findings From the Center for Research and Education on Aging and Technology Enhancement (CREATE)," Psychol Aging, vol. 21, no. 2, pp. 333-352, Jun. 2006, doi: 10.1037/08827974.21.2.333.

[5] M. G. Morris and V. Venkatesh, "Age differences in technology adoption decisions: implications for a changing work force," Personnel Psychology, vol. 53, no. 2, pp. 375-403, 2000, doi: 10.1111/j.17446570.2000.tb00206.x.

[6] J. Clawson, J. A. Pater, A. D. Miller, E. D. Mynatt, and L. Mamykina, "No longer wearing: Investigating the abandonment of personal health-tracking technologies on craigslist," 2015 , pp. 647-658, doi: 10.1145/2750858.2807554.

[7] D. A. Epstein, M. Caraway, C. Johnston, A. Ping, J. Fogarty, and S. A. Munson, "Beyond abandonment to next steps: understanding and designing for life after personal informatics tool use," in Proceedings of the 2016 CHI Conference on Human Factors in Computing Systems, New York, NY, USA, 2016, pp. 1109-1113, doi: 10.1145/2858036.2858045.

[8] D. A. Epstein, A. Ping, J. Fogarty, and S. A. Munson, "A lived informatics model of personal informatics," in Proceedings of the 2015 ACM International Joint Conference on Pervasive and Ubiquitous Computing UbiComp '15, Osaka, Japan, 2015, pp. 731-742, doi: 10.1145/2750858.2804250.

[9] A. Lazar, C. Koehler, J. Tanenbaum, and D. H. Nguyen, "Why we use and abandon smart devices," in Proceedings of the 2015 ACM International Joint Conference on Pervasive and Ubiquitous Computing, New York, NY, USA, 2015, pp. 635-646, doi: 10.1145/2750858.2804288.

[10] A. Cook and S. M. Hussey, Assistive technologies principles and practice, 2nd ed. Mosby: St. Louis, 2002.

[11] C. Doukas, V. Metsis, E. Becker, Z. Le, F. Makedon, and I. Maglogiannis, "Digital cities of the future: Extending@ @ome assistive technologies for the elderly and the disabled," Telematics and Informatics, vol. 28, no. 3, pp. 176-190, Aug. 2011, doi: 10.1016/j.tele.2010.08.001.

[12] R. Yared, H. K. Mallat, and B. Abdulrazak, "Ambient technology to support elderly people in outdoor risk situations," in Information and Communication Technologies for Ageing Well and e-Health, 2015, pp. 35-56.

[13] R. Yared and B. Abdulrazak, "Ambient Technology to Assist Elderly People in Indoor Risks," Computers, vol. 5, no. 4, p. 22, Dec. 2016, doi: 10.3390/computers5040022.

[14] S. Yusif, J. Soar, and A. Hafeez-Baig, "Older people, assistive technologies, and the barriers to adoption: A systematic review," International Journal of Medical Informatics, vol. 94, pp. 112-116, Oct. 2016, doi: 10.1016/j.ijmedinf.2016.07.004. 
[15] S. Federici, F. Meloni, and S. Borsci, "The abandonment of assistive technology in Italy: a survey of National Health Service users," Eur J Phys Rehabil Med, vol. 52, no. 4, pp. 516-526, Aug. 2016.

[16] B. Phillips and H. Zhao, "Predictors of assistive technology abandonment," Assist Technol, vol. 5, no. 1, pp. 36-45, 1993, doi: 10.1080/10400435.1993.10132205.

[17] A. T. Sugawara, V. D. Ramos, F. M. Alfieri, and L. R. Battistella, "Abandonment of assistive products: assessing abandonment levels and factors that impact on it," Disability and Rehabilitation: Assistive Technology, vol. 13, no. 7, pp. 716-723, Oct. 2018, doi: 10.1080/17483107.2018.1425748.

[18] M. J. Scherer and S. Federici, "Why people use and don't use technologies: Introduction to the special issue on assistive technologies for cognition/cognitive support technologies," NeuroRehabilitation, vol. 37, no. 3, pp. 315-319, Jan. 2015, doi: 10.3233/NRE151264.

[19] M. J. Scherer, C. Sax, A. Vanbiervliet, L. A. Cushman, and J. V. Scherer, "Predictors of assistive technology use: The importance of personal and psychosocial factors," Disability and Rehabilitation, vol. 27, no. 21, pp. 1321-1331, Jan. 2005, doi: 10.1080/09638280500164800.

[20] K. A. Mackintosh et al., "Parental perspectives of a wearable activity tracker for children younger than 13 years: Acceptability and usability study," JMIR mHealth and uHealth, vol. 7, no. 11, p. e13858, 2019, doi: 10.2196/13858.

[21] N. D. Ridgers et al., "Wearable activity tracker use among australian adolescents: Usability and acceptability study," JMIR mHealth and uHealth, vol. 6, no. 4, p. e86, 2018, doi: 10.2196/mhealth.9199.

[22] K. R. Evenson, M. M. Goto, and R. D. Furberg, "Systematic review of the validity and reliability of consumer-wearable activity trackers," Int J Behav Nutr Phys Act, vol. 12, Dec. 2015, doi: 10.1186/s12966-015-0314-1.

[23] J. K. Carroll, A. Moorhead, R. Bond, W. G. LeBlanc, R. J. Petrella, and K. Fiscella, "Who uses mobile phone health apps and does use matter? A secondary data analytics approach," Journal of Medical Internet Research, vol. 19, no. 4, p. e125, Apr. 2017, doi: 10.2196/jmir.5604.

[24] A. Kononova et al., "The use of wearable activity trackers among older adults: Focus group study of tracker perceptions, motivators, and barriers in the maintenance stage of behavior change," JMIR Mhealth Uhealth, vol. 7, no. 4, p. e9832, Apr. 2019, doi: 10.2196/mhealth.9832.

[25] L. Li, W. Peng, A. Kononova, M. Bowen, and S. R. Cotten, "Factors associated with older adults' longterm use of wearable activity trackers," Telemedicine and e-Health, Sep. 2019, doi: 10.1089/tmj.2019.0052.

[26] C. Maher, J. Ryan, C. Ambrosi, and S. Edney, "Users' experiences of wearable activity trackers: A crosssectional study," BMC Public Health, vol. 17, p. 880, Nov. 2017, doi: 10.1186/s12889-017-4888-1.

[27] E. D. de Bruin, A. Hartmann, D. Uebelhart, K. Murer, and W. Zijlstra, "Wearable systems for monitoring mobility-related activities in older people: a systematic review," Clin Rehabil, vol. 22, no. 10-11, pp. 878-895, Oct. 2008, doi: $10.1177 / 0269215508090675$.

[28] B. Williamson, T. Aplin, D. de Jonge, and M. Goyne, "Tracking down a solution: exploring the acceptability and value of wearable GPS devices for older persons, individuals with a disability and their support persons," Disability and Rehabilitation: Assistive Technology, vol. 12, no. 8, pp. 822-831, Nov. 2017, doi: 10.1080/17483107.2016.1272140.

[29] J. A. Lenker and V. L. Paquet, "A review of conceptual models for assistive technology outcomes research and practice," Assistive Technology, vol. 15, no. 1, pp. 1-15, Jun. 2003, doi: 10.1080/10400435.2003.10131885.

[30] M. J. Scherer, G. Craddock, and T. Mackeogh, "The relationship of personal factors and subjective wellbeing to the use of assistive technology devices," Disability and Rehabilitation, vol. 33, no. 10, pp. 811-817, Jan. 2011, doi: 10.3109/09638288.2010.511418.

[31] J. J. Annesi and P. H. Johnson, "Theory-based psychosocial factors that discriminate between weight-loss success and failure over 6 months in women with morbid obesity receiving behavioral treatments," Eating and Weight Disorders - Studies on Anorexia, Bulimia and Obesity, vol. 20, no. 2, pp. 223-232, Jun. 2015, doi: 10.1007/s40519-014-0159-7.

[32] S. Hermsen, J. Moons, P. Kerkhof, C. Wiekens, and M. De Groot, "Determinants for sustained use of an activity tracker: Observational study," JMIR mHealth and uHealth, vol. 5, no. 10, p. e164, Oct. 2017, doi: 10.2196/mhealth.7311.

[33] A. E. Bauman et al., "Correlates of physical activity: Why are some people physically active and others not?," The lancet, vol. 380, no. 9838, pp. 258-271, 2012.

[34] A. A. Eyler, R. C. Brownson, R. J. Donatelle, A. C. King, D. Brown, and J. F. Sallis, "Physical activity social support and middle- and older-aged minority women: results from a US survey," Social Science \& Medicine, vol. 49, no. 6, pp. 781-789, Sep. 1999, doi: 10.1016/S0277-9536(99)00137-9.

[35] B. N. Uchino, Social support and physical health: Understanding the health consequences of relationships. New Haven, US: Yale University Press, 2004.

[36] E. J. Lyons, Z. H. Lewis, B. G. Mayrsohn, and J. L. Rowland, "Behavior change techniques implemented in electronic lifestyle activity monitors: A systematic content analysis," J. Med. Internet Res., vol. 16, no. 8, p. e192, Aug. 2014, doi: 10.2196/jmir.3469.

[37] K. Mercer, M. Li, L. Giangregorio, C. Burns, and K. Grindrod, "Behavior change techniques present in wearable activity trackers: A critical analysis," JMIR mHealth and uHealth, vol. 4, no. 2, p. e40, Apr. 2016, doi: 10.2196/mhealth.4461.

[38] C. Elsden, D. S. Kirk, and A. C. Durrant, "A quantified past: Toward design for remembering with personal informatics," Human-Computer Interaction, 
vol. 31, no. 6, pp. 518-557, Nov. 2016, doi: $10.1080 / 07370024.2015 .1093422$.

[39] D. Ledger and D. McCaffrey, "Inside Wearables Part 1: How behavior change unlocks long-term engagement," 2014.

https://medium.com/@endeavourprtnrs/insidewearable-how-the-science-of-human-behaviorchange-offers-the-secret-to-long-term-engagementa15b3c7d4cf3 (accessed Jul. 09, 2020).

[40] G. Shin, Y. Feng, M. H. Jarrahi, and N. Gafinowitz, "Beyond novelty effect: A mixed-methods exploration into the motivation for long-term activity tracker use," Jamia Open, vol. 2, no. 1, pp. 62-72, Apr. 2019, doi: 10.1093/jamiaopen/ooy048.

[41] M. de Bruin et al., "Self-regulatory processes mediate the intention-behavior relation for adherence and exercise behaviors," Health Psychol, vol. 31, no. 6, pp. 695-703, Nov. 2012, doi: 10.1037/a0027425.

[42] S. Huisman, S. Maes, V. J. De Gucht, M. Chatrou, and H. R. Haak, "Low goal ownership predicts drop-out from a weight intervention study in overweight patients with type 2 diabetes," Int J Behav Med, vol. 17, no. 3, pp. 176-181, Sep. 2010, doi: 10.1007/s12529-009-9071-3.

[43] C. Fan, J. Forlizzi, and A. Dey, "Considerations for technology that support physical activity by older adults," in Proceedings of the 14th international ACM SIGACCESS conference on Computers and accessibility, 2012, pp. 33-40, Accessed: Jun. 28, 2017. [Online]. Available: http://dl.acm.org/citation.cfm?id=2384923.

[44] C. Courtois and P. Verdegem, "With a little help from my friends: An analysis of the role of social support in digital inequalities," New Media \& Society, vol. 18, no. 8, pp. 1508-1527, Sep. 2016, doi: $10.1177 / 1461444814562162$.

[45] A. J. A. M. van Deursen and J. A. G. M. van Dijk, "Using the Internet: Skill related problems in users' online behavior," Interacting with Computers, vol. 21, no. 5-6, pp. 393-402, Dec. 2009, doi: 10.1016/j.intcom.2009.06.005.

[46] H.-Y. S. Tsai, R. Shillair, and S. R. Cotten, "Social support and 'playing around': An examination of how older adults acquire digital literacy with tablet computers," J Appl Gerontol, vol. 36, no. 1, pp. 2955, Jan. 2017, doi: 10.1177/0733464815609440.

[47] T. Fritz, E. M. Huang, G. C. Murphy, and T. Zimmermann, "Persuasive technology in the real world: A study of long-term use of activity sensing devices for fitness," 2014, pp. 487-496, doi: 10.1145/2556288.2557383.

[48] S. Consolvo, K. Everitt, I. Smith, and J. A. Landay, "Design requirements for technologies that encourage physical activity," in Proceedings of the SIGCHI Conference on Human Factors in Computing Systems, New York, NY, USA, 2006, pp. 457-466, doi: 10.1145/1124772.1124840.

[49] J. J. Lin, L. Mamykina, S. Lindtner, G. Delajoux, and H. B. Strub, 'Fish'N'Steps: Encouraging physical activity with an interactive computer game," in Proceedings of the 8th International Conference on Ubiquitous Computing, Berlin, Heidelberg, 2006, pp. 261-278, doi: 10.1007/11853565_16.

[50] P. C. Shih, K. Han, E. S. Poole, M. B. Rosson, and J. M. Carroll, "Use and adoption challenges of wearable activity trackers," iConference 2015 Proceedings, 2015, Accessed: Dec. 29, 2016. [Online]. Available: https://www.ideals.illinois.edu/handle/2142/73649.

[51] A. Bhattacherjee, "Understanding information systems continuance: An expectation-confirmation mode," MIS Quarterly, vol. 25, no. 3, pp. 351-370, 2001, doi: 10.2307/3250921.

[52] M. Jaana and G. Pare, "Use of mobile health technologies for self-tracking purposes among seniors: A comparison to the general adult population in Canada," in Proceedings of the 53rd Hawaii International Conference on System Sciences (2020), Hawaii, Jan. 2020, pp. 3760-3769, doi: 10.24251/HICSS.2020.460.

[53] K.-J. Brickwood, G. Watson, J. O'Brien, and A. D. Williams, "Consumer-based wearable activity trackers increase physical activity participation: Systematic review and meta-analysis," JMIR mHealth and uHealth, vol. 7, no. 4, p. e11819, 2019, doi: 10.2196/11819.

[54] S. J. Hartman, S. H. Nelson, and L. S. Weiner, "Patterns of Fitbit use and activity levels throughout a physical activity intervention: Exploratory analysis from a randomized controlled trial," JMIR mHealth and uHealth, vol. 6, no. 2, p. e29, Feb. 2018, doi: 10.2196/mhealth.8503.

[55] J. Y.-W. Liu, P. P.-K. Kor, C. P.-Y. Chan, R. Y.-C. Kwan, and D. Sze-Ki, "The effectiveness of a wearable activity tracker (WAT)-based intervention to improve physical activity levels in sedentary older adults: A systematic review and meta-analysis," Archives of Gerontology and Geriatrics, vol. 91, p. 104211, Nov. 2020, doi: 10.1016/j.archger.2020.104211.

[56] T. L. Mitzner et al., "Older adults talk technology: Technology usage and attitudes," Computers in Human Behavior, vol. 26, no. 6, pp. 1710-1721, Nov. 2010, doi: 10.1016/j.chb.2010.06.020.

[57] A. Puri, B. Kim, O. Nguyen, P. Stolee, J. Tung, and J. Lee, "User acceptance of wrist-worn activity trackers among community-dwelling older adults: Mixed method study," JMIR Mhealth Uhealth, vol. 5, no. 11, p. e173, Nov. 2017, doi: 10.2196/mhealth.8211.

[58] S. J. Czaja, "Long-term care services and support systems for older adults: The role of technology," Am Psychol, vol. 71, no. 4, pp. 294-301, Jun. 2016, doi: 10.1037/a0040258.

[59] P. Khosravi and A. H. Ghapanchi, "Investigating the effectiveness of technologies applied to assist seniors: A systematic literature review," Int J Med Inform, vol. 85, no. 1, pp. 17-26, Jan. 2016, doi: 10.1016/j.ijmedinf.2015.05.014. 